\title{
A study on the Contamination of Microbial in a Geothermal Exchanger Pipe by Lab-experiment
}

\author{
Hyuntae Kim ${ }^{1, *}$, Makoto Koganei ${ }^{2}$ \\ ${ }^{1}$ Assistant Prof., Graduate School of Sciences and Technology for Innovation, Yamaguchi University, Japan \\ ${ }^{2}$ Prof., Graduate School of Sciences and Technology for Innovation, Yamaguchi University, Japan
}

\begin{abstract}
Recently, since global warming has continued, reduction of greenhouse gas CO2 is required. $\mathrm{CO} 2$ emissions in the civil area sectors in Japan account for approximately $30 \%$, which is about one-third of the whole of Japan. Therefore, reduction of energy consumption in houses, office and etc. is obviously required. In order to reduce energy consumption of buildings, high-efficiency of machinery, high-insulation, high-airtightness, and use of renewable energy are mentioned. Especially, use of geothermal energy which has almost constant temperature throughout the year has been drawing attention. However, in case of geothermal ventilation system, a fungi, dust, pollen and etc. derived from the outside air are taken directly into the pipe. It is concerned that they cause contamination inside the pipes and breeding of molds, which may lead to odors. The present study analyzed the contamination degree of the pipe surface in the geothermal ventilation system and proposed cleaning frequency in the pipe, cleaning method and so on.
\end{abstract}

\section{Introduction}

Today, as global warming progresses, reduction of greenhouse gas $\mathrm{CO}_{2}$ is required. $\mathrm{CO}_{2}$ emission standards for fiscal 2016 in Japan are about 34\% in the civilian sector, and approximately one-third of the total emissions comes from the civilian sector ${ }^{1)}$. Therefore, energy reduction in houses and buildings is required. The use of renewable energy plays a crucial role in reducing $\mathrm{CO}_{2}$ emissions. However, wind and sunlight are easily affected by the weather so it is difficult to secure energy steadily. Therefore, underground heat utilization which is almost constant throughout the year has been drawing attention ${ }^{2}$. Underground heat utilization includes heat pump geothermal system, air circulation, heat conduction, heat pipes, and so on ${ }^{2}$.

The current study targeted to use the geothermal ventilation system, which introduces outside air into the heat exchange pipe buried in the ground and takes the air cooled in the heat exchange pipe into the room. Since this system sends outside air to the room as it is, it plays as a role of ventilation as well as heat exchange of air. However, floating fungi, dust, pollen, etc. in the outdoor air are sent directly to the heat exchange pipe, and as a result, the inside of the heat exchange pipe is contaminated by the pollutants derived from the outside air. In addition, there is a concern that growth of microorganisms due to dew condensation on the pipe surface. Therefore, it is necessary to propose a healthy/hygienic method in terms of using the geothermal ventilation system.

This study aimed to measure the contamination concentration of the pipe surface in the geothermal ventilation system and propose the cleaning methods inside the underground pipe and the frequency of cleaning.

\section{Outline of Experiment}

Figure 1 shows the plan view of the laboratory, and Figure 2 demonstrates a sectional view of the laboratory A-A'. The experimental apparatus is installed in $\mathrm{Y}$ laboratory. The size of laboratory is $7300 \mathrm{~mm} \times 7000$ $\mathrm{mm}$, and the height of the ceiling is $2500 \mathrm{~mm}$. The entrance of the experimental apparatus was set outside the window so that the outside air was taken into the apparatus as it was.

\subsection{Experimental Device}

Figure 3 shows the detailed cross section of the experimental setup. Figure 4 shows the photograph of the experimental apparatus. The experimental apparatus is a model assuming a geothermal ventilation system. The experimental apparatus was manufactured using a heat insulating material with a thickness of $30 \mathrm{~mm}$, and the inside of the apparatus was finished with aluminum tape. The aluminum plate on the measurement surface is $250 \mathrm{~mm} \times 2000 \mathrm{~mm} \times 3 \mathrm{~mm}$ in size. In order to reproduce the surface temperature of the underground pipe, the aluminum plate is cooled by the cooling circulation device. The cooling water temperature is $17^{\circ}$ C. A fan is attached to the tip of the experimental apparatus so that the outside air is sucked in. The air volume is $550 \mathrm{~m}^{3} / \mathrm{h}$. The size of the duct is $280 \mathrm{~mm} \times$

* Corresponding author: hyuntae@yamaguchi-u.ac.jp 
$300 \mathrm{~mm}$ in inner diameter, and the wind speed in the duct is about $1.8 \mathrm{~m} / \mathrm{s}$.

\subsection{Measurement Method}

Table 1 explains the measuring device in detail. In order to confirm the contamination situation of the underground heat pipe surface, the contamination level was measured by ATP measurement, and mold concentration on the pipe surface was measured by using a stamp type agar medium. In addition, the mold concentration in the outside air was measured. ATP uniformly wipes off the range of $5 \mathrm{~cm} \times 10 \mathrm{~cm}$. The assessment of contamination degree is performed by converting the measurement result into area per $100 \mathrm{~cm}^{2}$.

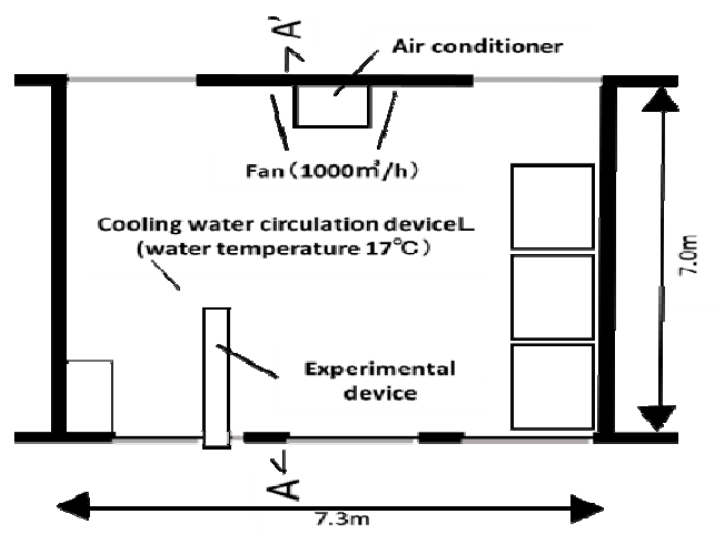

Fig 1. The plan view of the laboratory

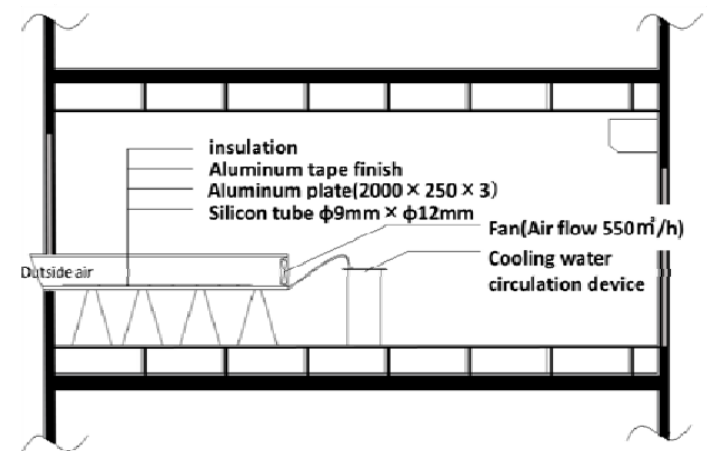

Fig 2. The sectional view of laboratory A-A'

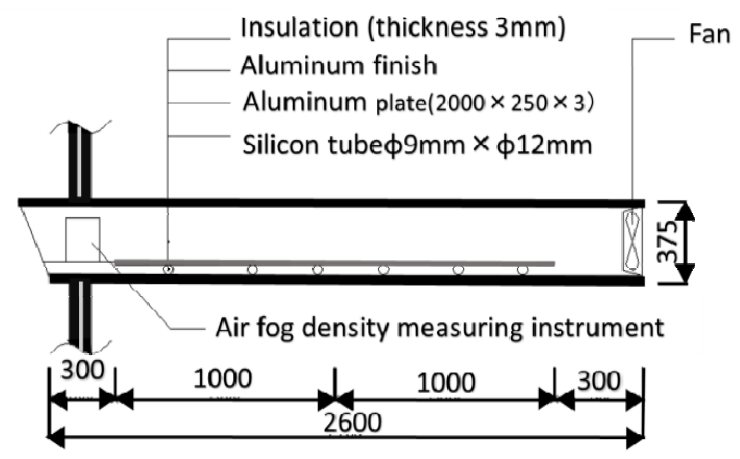

Fig 3. The detailed cross section of the experimental setup
Table 2 shows the label of contamination concentration. Stamp type agar medium (PDA) was used for measuring mold concentration on the surface of aluminum plate. The condition for culturing is at 28 degrees for 3 days. In order to lower the initial concentration of the aluminum plate, the aluminum plate was thoroughly washed and sterilized. Figure 5 presents the photograph of cultivation and ATP concentration apparatus. Figure 6 shows the point of measurement. Here is how the measurement was performed : leave both ends $2.5 \mathrm{~cm}$ each; and then divide the square into $5 \mathrm{~cm} \times 5 \mathrm{~cm}$ squares by 40 horizontal points and 4 vertical sections to make a total of 160 measurement points. The range to be measured at one time is four squares of $5 \mathrm{~cm} \times 5 \mathrm{~cm}$. ATP was measured in the range of $10 \mathrm{~cm} \times 5 \mathrm{~cm}$ on the left side, and on the other hand, the surface mold density was measured twice in the range of $5 \mathrm{~cm} \times 5 \mathrm{~cm}$ on the right side. The aluminum plate was divided into the three directions of $\mathrm{A}, \mathrm{B}$ and $\mathrm{C}$ in the direction of the long side, and then measurement was carried out at each one place, and the result was obtained as an average value. In addition, the fresh mold concentration and temperature and humidity at the entrance and exit of the outside air were measured. For the measurement, the day on which the aluminum plate was sterilized was taken as the 0th day, and the measurement was carried out for 4 days from this day.
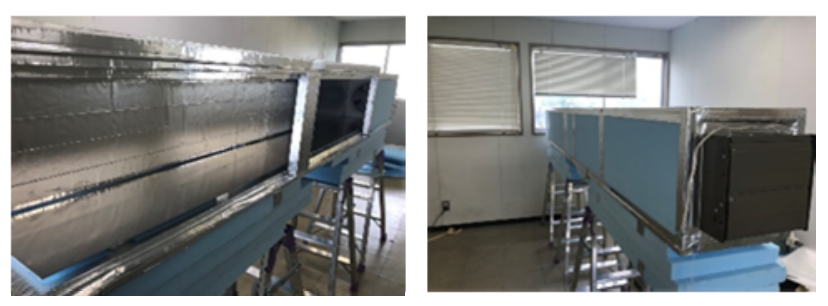

Fig.4. the photograph of the experimental apparatus

Table 1. The measuring device

\begin{tabular}{|c|c|}
\hline Measurement item & Details \\
\hline Temperature and humidity & Espec corp. RS-14 \\
\hline Airborne mold concentration & IDC-500B \\
\hline ATP concentration & $\begin{array}{c}\text { System SURE Plus } \\
\text { Supersnap }\end{array}$ \\
\hline Surface mold concentration & Stamp type PDA agar \\
\hline Cooling system & LTC-1200 $\alpha$ \\
\hline Mold cultivation & Incubator \\
\hline
\end{tabular}

Table 2. The label of contamination concentration

\begin{tabular}{|c|c|c|}
\hline Level & \multicolumn{2}{|c|}{ Ensure $\times$ Supersnap } \\
\hline I & Extremely clean & $0 \sim 100$ \\
\hline II & Very clean & $110 \sim 300$ \\
\hline III & Usually & $310 \sim 800$ \\
\hline IV & Somewhat dirty & $810 \sim 2000$ \\
\hline V & dirty & $2010 \sim 5000$ \\
\hline VI & Very dirty & $5010 \sim 10000$ \\
\hline VII & Extremely dirty & $10010 \sim$ \\
\hline
\end{tabular}


On day 0 , measurements were taken every 3 hours from 9 o'clock, and changes over time were observed. Measurements on days 1, 2, and 3 were made once in the morning.

\section{Results}

The value unit of ATP is RLU (Relative Light Unit). ATP present in the cell emits light by reacting with the enzyme luciferase or the like ${ }^{3)}$. Immediately after sterilization, the measured ATP was 5 to 8 [RLU], and it was evaluated as "extremely clean". The measurement result is the average value of the 3 measurement points. Figure 7 shows the time-course changes of ATP measured values. It was "extremely clean" 3 hours after sterilization. However, it turned out that the surface of the aluminum was gradually contaminated over the time. The concentration of ATP after 24 hours is about 2500 [RUL], which is greatly increased. From around 3rd day, it seems that the degree of pollution becomes steady state. When the contamination level is evaluated by using ATP, the 0th day is "ordinary." After the first day, it is evaluated as "dirty".

Figure 8 shows the mold concentration of the surface. Surface mold concentration was counted by the number of mold colonies cultured for 3 days. The number of molds on the aluminum surface was found to be increased until the second day. The maximum value of the number of surface molds on the aluminum plate was $35000\left[\mathrm{CFU} / \mathrm{m}^{2}\right]$. However, the number of colonies on the third day is slightly decreased. It is thought that fluctuation of mold concentration in the outside air affected this result. Figure 9 shows the concentration of mold in the outside air. The concentration of mold in the outside air was 200 to $600\left[\mathrm{CFU} / \mathrm{m}^{3}\right]$. The mold concentration at day 0 and day 1 were measured high. However, the mold concentration at days 2 and 3 were measured low. This result seems to have influenced the surface mold concentration on the third day.
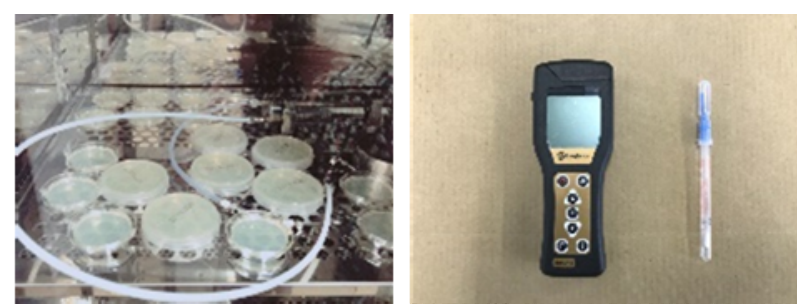

The study results demonstrate that the surface of the geothermal ventilation system needs to be cleaned once every three days.

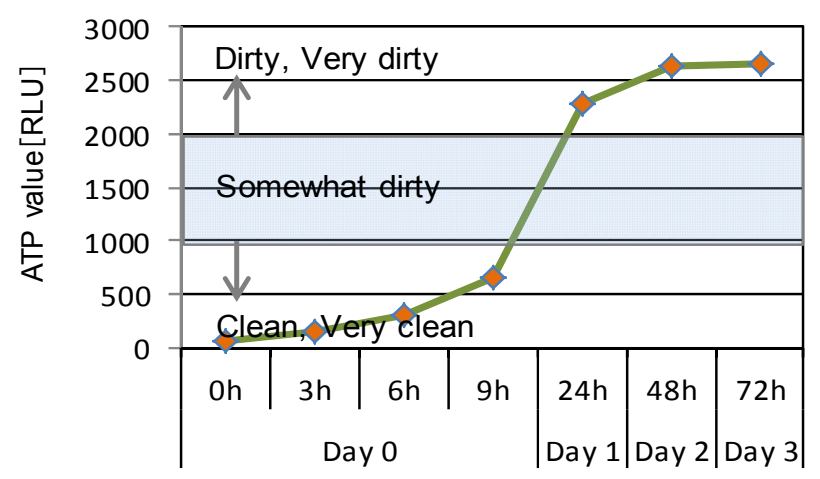

Fig.7. The time-course change of ATP measured values

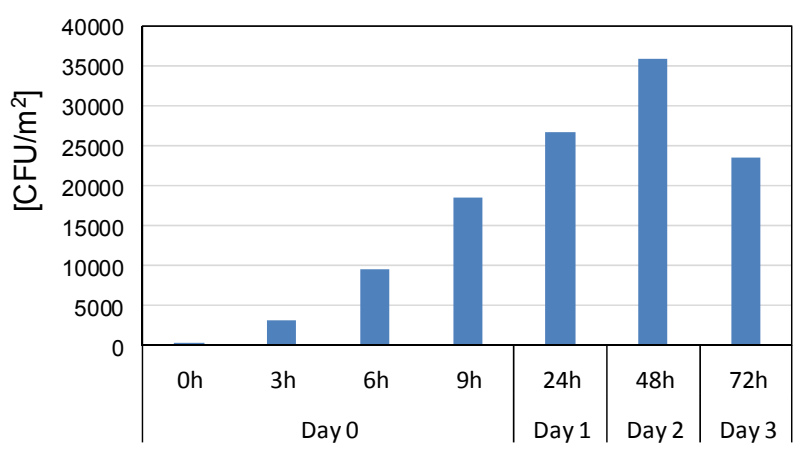

Fig.8. The mold concentration of the surface

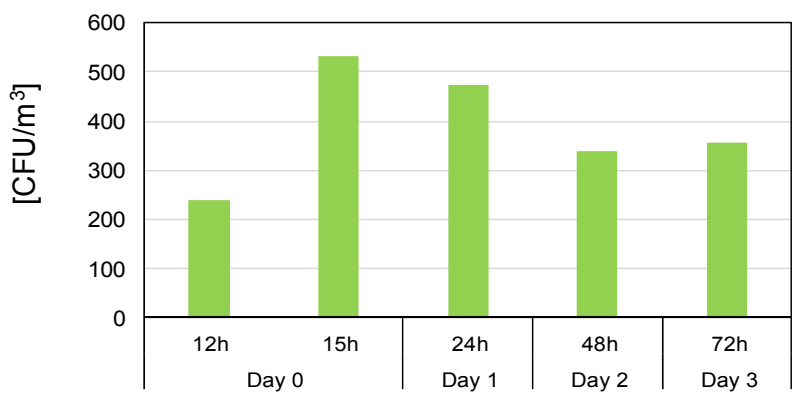

Fig.9. The concentration of mold in the outside air

Fig.5. The photo of cultivation and ATP apparatus

fan $\longrightarrow$

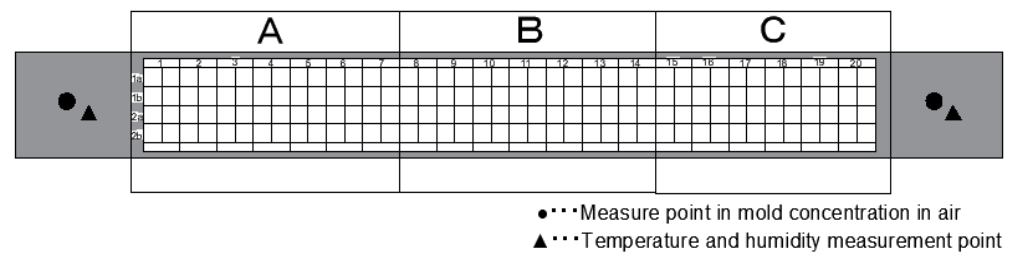

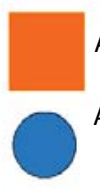

ATP measurement

Agar medium stamp measurement

Fig 6. The point of measurement 


\section{Conclusions}

This study analyzed the contamination degree of the pipe surface in the geothermal ventilation system and proposed cleaning frequency in the pipe and cleaning method. The study results are as follows:

1) The study found that the surface of aluminum was gradually contaminated by the time changes. It can be seen that the concentration of ATP after 24 hours is about 2500 [RUL], which is greatly increased. From around 3rd day, it seems that the degree of pollution becomes steady state.

2) The concentration of mold on the aluminum surface was found to be increased until the 2 nd day. The maximum value of the concentration of surface mold on the aluminum was $35000\left[\mathrm{CFU} / \mathrm{m}^{2}\right]$.

3) The concentration of mold in the outside air was 200 to $600\left[\mathrm{CFU} / \mathrm{m}^{3}\right]$. The mold concentration at day 0 and day 1 were measured high. But, the mold concentration at days 2 and 3 were measured low. This result seems to have influenced the surface mold concentration on the third day.

4) From the results above, it is considered that the surface of the geothermal ventilation system needs to be cleaned once every day.

\section{Acknowledgments}

This work was supported by Grant-in-Aid for Scientific Research (C) in 2018.

\section{References}

1. A. Mecke, I. Lee, J.R. Baker jr., M.M. Banaszak Holl, B.G. Orr, Eur. Phys. J. E 14, 7 (2004)

2. M. Ben Rabha, M.F. Boujmil, M. Saadoun, B. Bessaïs, Eur. Phys. J. Appl. Phys. (to be published)

3. Luigi T. De Luca, Propulsion physics (EDP Sciences, Les Ulis, 2009)

4. F. De Lillo, F. Cecconi, G. Lacorata, A. Vulpiani, EPL, 84 (2008) 